\title{
Patrones de Deglución y Metodología de Evaluación. Una Revisión de la Literatura
}

\author{
Swallowing Patterns and Evaluation Methodology. A Review of the Literature \\ Priscila Chuhuaicura'; Giannina Álvarez'; María Florencia Lezcano ${ }^{1,2}$; Alain Arias ${ }^{1,2,3}$; \\ Fernando Jose Dias ${ }^{1,2}$ \& Ramón Fuentes ${ }^{1,2}$
}

CHUHUAICURA, P.; ÁlVAREZ, G.; LEZCANO, M. F.; ARIAS, A.; DIAS, F. J. \& FUENTES, R. Patrones de deglución y metodología de evaluación. Una revisión de la literatura. Int. J. Odontostomat., 12(4):388-394, 2018.

RESUMEN: La deglución es una función indispensable para el ser humano, es una actividad compleja que necesita de la participación y coordinación de múltiples estructuras. Actualmente existen diferentes métodos para evaluar este proceso, sin embargo, la capacidad de describir el comportamiento de las estructuras en sus tres dimensiones es limitada. Se ha demostrado que el movimiento lingual, es clave para la formación del bolo alimenticio y la propulsión de este a la orofaringe. Es así como se han identificado patrones de movimiento lingual a través de diferentes técnicas. El objetivo de esta revisión es reportar las diferentes técnicas de evaluación de la deglución e identificar los patrones que establece cada una de ellas. Se realizó una revisión de la literatura en distintas bases de datos, a través de una estrategia de búsqueda sistematizada. Se utilizaron las palabras clave "Deglutition" "Swallowing" "Movement Tracking" "evaluation" y "patterns" en inglés y español. Se incluyeron estudios sin límite en la fecha de publicación, realizados en humanos, en idioma inglés, español y portugués. Se encontraron diferentes técnicas para evaluar la deglución, videofluoroscopía, endoscopía, articulografía electromagnética, electromiografía, tomografía computarizada, manometría, electropalatografía y ultrasonido. Entre las cuales la videofluoroscopía es una de las más utilizadas, con esta técnica se ha intentado describir patrones del movimiento durante la deglución, al igual que la articulografía electromagnética. Por otro lado, la electromiografía ha descrito patrones de deglución, pero en relación a la contracción y relajación de ciertos grupos musculares. Existen diferentes métodos para evaluar la deglución, cada una de estas técnicas ha permitido dilucidar de mejor forma este proceso, no obstante, es necesario una mayor cantidad de estudios comparativos que permitan resolver las dudas sobre la validez y la eficacia de las técnicas descritas.

PALABRAS CLAVE: deglución, movimiento lingual, patrones.

\section{INTRODUCCIÓN}

Se ha reportado que funciones básicas como la masticación, deglución, respiración y producción del habla, están incorporadas dentro de un sistema orofacial quien se mantiene en funcionamiento gracias a un correcto desarrollo y mantenimiento de las estructuras involucradas (Engelke et al., 2010; Knösel et al., 2011).

La deglución es una de las funciones más complejas y necesarias para el desarrollo de la vida humana y requiere de la actividad de múltiples regiones cerebrales y de un riguroso control neuromuscular de estructuras como: cavidad oral, faringe y esófago (Jestrovic et al., 2016). El estudio de la deglución ha ido cobrando cada vez mayor relevancia, esto porque las estructuras que participan en este proceso requieren de un desarrollo y coordinación adecuados para transportar el bolo alimenticio desde la cavidad oral hasta el estómago sin permitir la entrada de ninguna sustancia a la vía aérea (Dodds et al., 1989). En condiciones normales, la cavidad oral es un sistema que forma un perfecto equilibrio entre los dientes, la lengua y los tejidos blandos periorales. El equilibrio de los tejidos blandos forma una matriz capsular cerrada, que genera diferencias de presión con el ambiente atmosférico (Fuentes et al., 2015).

La deglución se ha dividido en cuatro fases, la primera es la fase preparatoria oral, que consiste en la formación del bolo alimenticio, mediante la trituración de los alimentos y la mezcla de estos con la saliva. La segunda etapa es la fase oral, aquí el bolo alimenticio mediante una combinación de movimientos linguales es transportado desde el dorso de la lengua hasta la entrada de la faringe. Ya en la tercera etapa que corresponde a la fase faríngea comienza con el pasaje del bolo ali-

\footnotetext{
${ }^{1}$ Centro de Investigación en Ciencias Odontológicas (CICO), Facultad de Odontología, Universidad de La Frontera, Temuco, Chile.

${ }^{2}$ Departamento de Odontología Integral Adultos, Facultad de Odontología, Universidad de La Frontera, Temuco, Chile.

${ }^{3}$ Universidad Adventista de Chile, Chillán, Chile.
} 
menticio desde la base de la lengua, a través del istmo de las fauces, hasta la pared faríngea posterior. El contacto del bolo contra la mucosa del paladar blando, faringe y epiglotis, actúa como estímulo de una serie de mecanismos reflejos cuya principal función es asegurar que el bolo llegue al esófago sin ingresar ni a la tráquea ni a la nasofaringe. La última es la fase esofágica que comienza inmediatamente después del pasaje del bolo alimenticio por el esfínter esofágico, y se caracteriza por las contracciones musculares que permiten el transporte del alimento a lo largo del esófago, que finalmente llevarán el bolo hasta el estómago (Chi-Fishman et al., 1998; Doods et al.).

Para evaluar la deglución existen diferentes técnicas, una de ellas es a través de un análisis subjetivo, donde se observan movimientos compensatorios de cabeza y cuello, y la contracción excesiva de músculos como el mentoniano. Debido a la dificultad de establecer parámetros objetivos, es que surgen métodos tales como videofluoroscopía, endoscopía, ultrasonido, articulografía electromagnética, electromiografía, tomografía computarizada, manometría y electropalatografía (Steele \& Van Lieshout, 2004, 2008, 2009) que permiten constatar cómo actúa la musculatura oral y la lengua durante la deglución. Entre estas técnicas se considera como gold estándar a la videofluroscopía. Sin embargo, su uso está siendo debatido, por la aparición de nuevas herramientas diagnósticas.

Pese a que en los últimos años ha incrementado el interés por analizar el proceso de deglución, existe poco consenso en la literatura sobre la determinación de los patrones que producen las estructuras involucradas, siendo el objetivo de esta revisión reportar las diferentes técnicas de evaluación de la deglución e identificar los patrones que establece cada una de ellas.

\section{MATERIAL Y MÉTODO}

Se realizó una revisión con búsqueda sistematizada en las siguientes bases de datos: Pubmed/ Medline, Web of Sciences, Science Direct, Scopus, Embase y SciELO.

Se utilizó una estrategia de búsqueda con las palabras clave "Deglutition" "Swallowing" "Movement Tracking" "evaluation" y "patterns" en inglés y español. Se incluyeron estudios sin límite en la fecha de publicación, realizados en humanos, en idioma inglés, español y portugués.
Se excluyeron del estudio cartas al editor, reportes de caso y estudios experimentales realizados en animales.

\section{RESULTADOS}

Tanto la búsqueda como la selección de los artículos analizados en este estudio está descrito en la Figura 1. Se encontraron 89 artículos directamente desde las bases de datos, además se realizó una búsqueda manual en la que se incluyeron 8 artículos. Se realizó una lectura inicial por título y resumen, donde se excluyeron 72 artículos de los cuales 61 eran duplicados, 8 artículos no presentaban relación con el tema de estudio y 3 eran reportes de caso. Finalmente se analizaron 25 artículos.

Evaluación de Patrones de Deglución. Distintos autores han analizado el comportamiento de la lengua y el paladar para establecer un patrón de deglución genérico, sin embargo, se ha demostrado que cada individuo tiene un patrón de deglución propio (MacAvoy et al., 2016).

En el año 1989 Doods et al. determinó dos patrones de deglución mediante la videofluoroscopia (VF), el patrón "tipper" se presenta cuando todo el bolo se encuentra sobre la lengua, necesitando sólo elevarse para propulsar el alimento hasta la faringe. En comparación al patrón "dipper" el bolo se encuentra en su mayoría debajo de la lengua al inicio de la deglución, por lo que se debe generar un movimiento lingual en forma de cuchara para que el alimento adopte a una posición supralingual, luego el desplazamiento del bolo hasta la faringe se realiza de la misma manera que en el patrón tipper. (Doods et al.,). Estos resultados fueron justificados por Bourdiol et al. (2014) quienes reportaron tres patrones de deglución utilizando articulografía electromagnética 2D , los primeros dos hicieron referencia al estudio descrito por Doods, sin embargo, se identificó un patrón adicional, en donde la mandíbula se encuentra en una posición inicial ligeramente elevada, luego tanto la mandíbula como el dorso lingual realizan un movimiento descendente en conjunto para que finalmente la lengua se eleve en su posición, realizando el impulso para desplazar el alimento hasta la faringe.

La electropalatografía también ha sido utilizada para determinar los patrones de movimiento entre el dorso de la lengua y el paladar duro, pero esta 


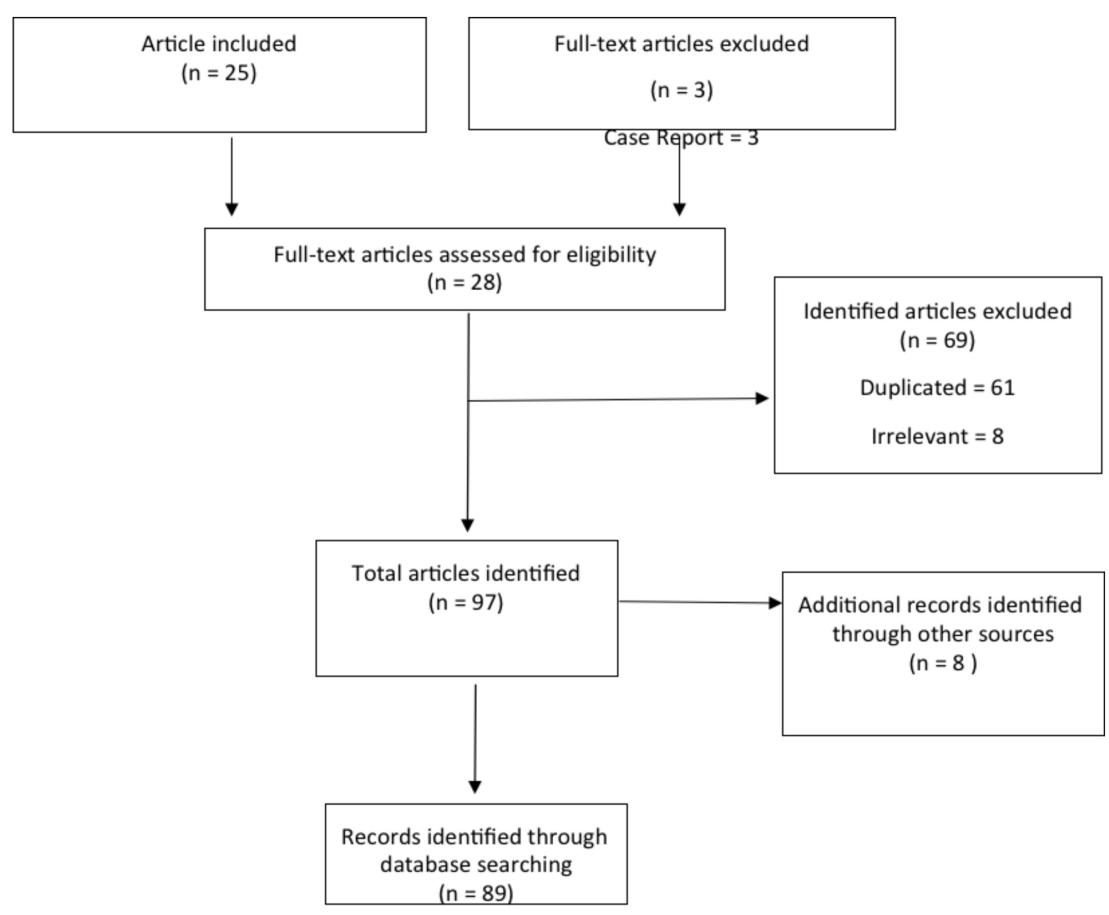

Fig. 1. Diagrama de flujo de la revisión sistematizada.

técnica no permite identificar que porción de la lengua entra en contacto con el paladar, además no permite plasmar el movimiento de la lengua antes o después del contacto con el paladar (Chi-Fishman \& Stone, 1996; Steel \& Van Lieshout, 2009).

A través de electromiografía (EMG) con electrodos de aguja concéntricos, Gokyigit et al. (2009) analizaron los músculos tiroaritenoideos (TA) y cricofaríngeos (CF), e investigaron el intervalo de tiempo entre el cierre glótico y la apertura el esfínter esofágico superior durante la deglución, basándose en el inicio de la actividad EMG y la relajación de los músculos descritos. En cuanto a la asociación entre TA-EMG y CF-EMG, se definieron tres patrones, el patrón A está definido cuando existe un retraso en la actividad electromiográfica del músculo TA, el patron $B$ es cuando existe una superposición entre la TAEMG y la CF-EMG, por último, en el patrón $C$ existe una aparición más temprana de la TA-EMG en relación al CF-EMG (Gokygit et al., 2009).

\section{Técnicas de Evaluación de la Deglución}

Videofluoroscopía. Esta técnica es una de las más utilizadas y además es considerada gold estándar para la evaluación de la deglución orofaringea. El procedimiento consiste en visualizar la deglución ya sea de saliva o alimento adicionando un medio de contraste radio-opaco, la imagen queda registrada en una cámara de video que está situada frente a una pantaIla radioscópica, esto permite analizar detenidamente el proceso. Dentro de las ventajas de este examen está la evaluación completa de todas las fases de la deglución, incluyendo el registro de movimientos compensatorios y del tamaño y consistencia del bolo alimenticio que maneja el paciente (Nazar et al., 2008). Sin embargo, estudios afirman que posee una capacidad limitada de valoración de los movimientos linguales y riesgo por exposición a radiación con el uso de bario (Steele \& Van Lieshout, 2004).

Endoscopía con fibra óptica. La endoscopía se ha utilizado como alternativa a la videofluoroscopia para el diagnóstico y tratamiento de disfagia en población adulta e infantil. A través de una cámara, muestra el estado de las estructuras anatómicas involucradas, antes y después de la deglución. Previo a la deglución, el endoscopio debe ubicarse en el paladar blando, entre el velo y la epiglotis, para visualizar la base de la lengua y evaluar la llegada del bolo alimenticio a la faringe (Nazar et al.). La ventaja es que no expone a radiación y permite la visualización de la anatomía y fisiología de la fase faríngea durante el proceso 
deglutorio sin embargo no logra reproducir la fase oral de la deglución (Logemann et al., 1998; Langmore, 2003). Es un examen versátil, portátil y seguro, generalmente bien tolerado, que permite evaluar e implementar maniobras compensatorias para permitir una correcta deglución (Langmore et al., 1991; Reynolds et al., 2016).

Articulografía electromagnética. Durante la última década el uso de la articulografía electromagnética ha ido en aumento, si bien en sus inicios se utilizó para estudiar los movimientos linguales durante la producción del habla. Actualmente se ha reportado su uso para medir los movimientos de la lengua durante la fase preparatoria y oral de la deglución. (Steel et al.) Pese a que el articulógrafo electromagnético no permite la visualización de tejidos blandos, ni de la forma o tamaño del bolo alimenticio, si proporciona una evaluación espacio temporal detallada de la lengua durante la deglución (Steel et al.; Bourdiol et al.).

Electromiografía. Ya que la deglución es esencialmente una actividad muscular, la electromiografía (EMG) también se ha utilizado para evaluar este proceso, principalmente se han publicado estudios de EMG de superficie con el uso de electrodos y EMG de punción con agujas. La EMG tiene un papel relevante en el estudio de la fases oral y faríngea del proceso deglutorio, ya que éste acto depende de la coordinación de más de 20 músculos (Padrós Serrat, 2004). Los grupos musculares más analizados son el masétero, músculos suprahioideos e infrahioideos, además algunos estudios reportan el comportaminto de músculos más profundos como tiroaritenoideos y cricofaríngeos (Goykigit et al.) Dentro de las ventajas permite identificar la duración e intensidad de la contracción además de evaluar la simetría y cuantificar la armonía de la contracción muscular entre pares de músculos del lado derecho e izquierdo del cuerpo (MacAvoy et al.).

Tomografía computarizada. La tomografía computarizada (CT) es una técnica relativamente nueva para el estudio de la deglución, es comúnmente utilizada para el diagnóstico de condiciones patológicas a través de imágenes transversales (Fujii et al., 2010), la CT convencional permite conseguir imágenes transversales de la orofaringe para analizar la mecánica deglutoria en 3D, dentro de sus limitaciones está la escasa resolución temporal para obtener imágenes rápidas sobre los movimientos producidos durante la deglución (Ergun et al., 1993). Actualmente con las nuevas tecnologías en CT las imágenes obte- nidas permiten escanear tanto la cavidad oral como la faringe, laringe y la porción superior del esófago con una adecuada resolución, facilitando el análisis del proceso deglutorio (Kobayashi et al. 2018). Fujii et al. utilizó el 320-Detector-Row Multislice CT para investigar morfología y análisis cinemáticos de la deglución, a través de las imágenes se pueden evaluar las estructuras que participan en la fase oral, faríngea y esofágica.

Manometría. La manometría también se ha empleado para determinar la actividad motora del esófago y de sus esfínteres. Se realiza a través de un tubo que contiene sensores de presión a varios intervalos (Rosen et al., 2018). Estos entregan información de la fuerza de propulsión faríngea, la coordinación faringoesofágica además de las contracciones y relajaciones apropiadas a nivel esofágico, esta técnica a diferencia de otras se enfoca en analizar desde el término de la fase faríngea hasta la fase esofágica. Dentro de las ventajas se considera la portabilidad del dispositivo y el mínimo posicionamiento del paciente. Si bien la manometría convencional presentaba ciertas limitaciones, el posterior desarrollo de la Manometría de alta resolución (HRM) proporcionó la capacidad de capturar e informar simultáneamente la presión generada durante la deglución (Park et al., 2017).

Electropalatografía. La electropalatografía es una técnica que permite determinar los contactos de la lengua con el paladar a través de la activación de electrodos que se encuentran en la superficie de una placa artificial acrílica adaptada para cada paciente (Fernández Planas, 2008). Para facilitar su estudio, la electropalatografía se ha dividido en cuatro estadíos, prepropulsión, propulsión, contacto completo y retirada (Padrós Serrat), que aluden a las fases oral y faríngea de la deglución. Además, compartimentar la placa en seis partes (frontal, central, posterior, lateral, medial y linea media) permite caracterizar los contactos linguopalatinos. Esta técnica se ha utilizado para examinar la ubicación, el patrón, y el momento de los contactos linguopalatales durante la fonoarticulación y los trastornos del habla además permite obtener información visual demostrando asertivamente la percepción sensorial oral alterada (Chi-Fishman et al.).

Ultrasonido. Se utilizan sonidos de alta frecuencia para producir imágenes dinámicas de los tejidos blandos, permite visualizar la relación temporal de los movimientos realizados por las distintas estructuras. Las ventajas de esta técnica se resumen en términos de costo del equipamiento, portabilidad, imágenes en 
tiempo real, confort para el paciente y seguridad para la salud (Rocha et al., 2015). De acuerdo a lo reportado por Ardakani esta técnica se puede implementar para diagnosticar con certeza las patologías de la deglución, ya que posibilita la visualización directa de los movimientos de la lengua en planos coronal y sagital. Por lo tanto, esta una técnica permite evaluar con precisión la fase preparatoria y oral de la deglución (Ardakani, 2006).

Presiones Intraorales. Las presiones intraorales han sido estudiadas, para establecer su influencia en el proceso de deglución (Hiss et al., 2001). De acuerdo al modelo biofuncional (Engelke et al.), en condiciones normales, la cavidad oral presenta un sistema de equilibrio entre los dientes, los tejidos blandos y la lengua, el equilibrio producido genera una matriz capsular cerrada y compuesta de compartimentos biofuncionales intraorales que generan diferencias de presión con el ambiente atmosférico, esta diferencia es conocida como presión intraoral (Fuentes et al.). Para la evaluación de las presiones intraorales, se han implementado técnicas con unos medidores de presión digital portátil conectado a dos sensores de presión relativa, así mismo se han utilizado transductores de presión en miniatura capaces de medir presiones absolutas a un aparato palatino de cromo-cobalto individualizado para cada paciente (Kieser et al., 2011; Fuentes et al.).

\section{DISCUSIÓN}

La presente revisión describió las técnicas utilizadas para evaluar la deglución, los patrones que establece cada metodología y aspectos de la deglución como la evaluación de las diferentes estructuras que participan en el proceso. De acuerdo a lo reportado en la literatura, durante años se ha intentado explicar el comportamiento de las estructuras involucradas durante el proceso de deglución. Para ello, algunos autores han establecido patrones para comprender el movimiento de la lengua, o para evaluar la apertura y cierre de la epiglotis en conjunto con la contracción de grupos musculares. Tanto la videofluoroscopía, endoscopía, articulografía electromagnética y electromiografía han sido las técnicas más utilizadas para identificar patrones en deglución.

Entre ellas la videofluoroscopia ha sido una de las técnicas más utilizadas, pero tiene ciertas limita- ciones, ya que es un examen complejo de ejecutar, requiere de un tiempo prolongado y el paciente se expone a radiación. Por esto se fomentó el desarrollo de nuevas metodologías para la evaluación de este proceso (Arteaga et al., 2006).

Existen estudios comparativos entre las distintas técnicas. Fattori et al. (2016) evaluó la videofluoroscopía, la endoscopía de fibra óptica y gammagrafía (técnica similar a la tomografía computarizada, pero menos conocida que emplea isótopos radioactivos para estudiar el funcionalismo de distintos órganos), se encontró que tanto la endoscopía como la gammagrafía poseen una adecuada sensibilidad diagnóstica y por lo tanto ambos exámenes pueden realizarse para el diagnóstico de patologías de la deglución. (Fattorri et al., 2016) Resultados similares se observaron en el estudio realizado por da Silva et al. (2010). Los autores concuerdan que es necesario desarrollar una mayor cantidad de estudios comparativos que permitan resolver las dudas sobre la validez y la eficacia de las técnicas descritas (Fattori et al.; da Silva et al.; Reynolds et al.; Logemann et al.).

Por otra parte, Steel et al. demostró que el Articulografía Electromagnética Mediosagital (EMMA) permite analizar el comportamiento de las estructuras orales durante la deglución. Esta técnica presenta ventajas principalmente en la cantidad de datos que logra procesar tanto en la cuantificación como en la precisión de movimientos linguales. En estudios posteriores del año 2007 y 2009 se analizaron los cambios relacionados con la edad en los movimientos de la lengua durante la deglución. Los datos obtenidos afirman existen patrones estereotipados específicos para el movimiento de la lengua, y que estos no varían con el envejecimiento ni con el género (Steel et al. 2004, 2007,2009 ). Bourdiol et al. identificó tres patrones que guardan directa relación a la posición de la lengua durante la deglución.

Respecto a las técnicas electromiográficas se han descrito diferentes metodologías de evaluación que, a diferencia de los otros estudios reportados anteriormente, examinan otras estructuras musculares. Zhu et al. (2017) analizaron el proceso de deglución en adultos sanos a través de electromiografía de superficie de alta densidad utilizando 96 electrodos ubicados en la región frontal del cuello, se realizaron diferentes pruebas de deglución tanto de líquidos como semisólidos. Por otro lado, Vaiman et al. (2004a,b) mediante la electromiografía de superficie analizó grupos musculares definidos utilizando 8 electrodos, las 
pruebas de deglución se realizaron con saliva y agua en diferentes volúmenes. Ambos autores concluyen que los métodos propuestos evalúan fisiológicamente las características dinámicas de la deglución (Vaiman et al., 2004a,b; Zhu et al.) No obstante, ambos estudios utilizaron metodologías distintas para llevar a cabo el mismo análisis, por lo que sería apropiado generar estudios que comparen la técnica gold estándar con la EMG, para cuantificar validez, sensibilidad y eficacia de las metodologías propuesta.

De acuerdo a lo planteado en los resultados, generalmente las técnicas de evaluación de la deglución analizan sólo una parte del proceso, es decir que permiten observar solo ciertas fases de la deglución. Tal es el caso del ultrasonido, la articulografía electromagnética y la medición de presiones intraorales que evalúan tanto la fase preparatoria y oral. (Steel et al.; Ardakani; Fuentes et al.) Por otro lado, la fase oral y faríngea se estudian a través de técnicas como la electropalatografía, electromiografía y tomografía computarizada multicorte (Padrós Serrat; Fujii et al.), la manometría evalúa parte de la fase faríngea y la fase esofágica (Park et al.). Sin embargo, se han descrito técnicas que solo observan una fase de la deglución, como es el caso de la endoscopía que analiza sólo la fase faríngea (Logemann et al.; Langmore), por último, la videofluoroscopía que permite evaluar todas las fases de la deglución (Nazar et al.). Por lo tanto, se estima que utilizar una sola técnica de evaluación, otorga al clínico una mirada parcial sobre el proceso deglutorio, siendo necesario utilizar varias técnicas simultáneamente para comprender a cabalidad el comportamiento de las estructuras. De los artículos analizados ninguno de ellos utilizó técnicas simultáneas para evaluar la deglución, pero si, se constataron estudios comparativos que fueron mencionados con anterioridad.

\section{CONCLUSIONES}

Existen diversas técnicas y metodologías para el estudio de la deglución, cada una de ellas presenta ventajas y desventajas que el clínico debe identificar para ofrecer el diagnóstico y tratamiento más adecuado para el paciente. La mayoría de las técnicas analizadas propone distintos patrones de comportamiento de las estructuras de la mecánica deglutoria, además no todas las técnicas evalúan la misma fase de la deglución. Para obtener el diagnóstico más certero se precisa de un examen minu- cioso que implemente anamnesis y técnicas simultáneas, que permitan reconocer los trastornos de la deglución y proponer tratamientos acordes a las necesidades de los pacientes.

CHUHUAICURA, P.; ÁLVAREZ, G.; LEZCANO, M. F.; ARIAS, A.; DIAS, F. J. \& FUENTES, R. Swallowing patterns and evaluation methodology. A review of the literature. Int. J. Odontostomat., 12(4):388-394, 2018.

ABSTRACT: Swallowing is a critical function for the human being. It is a complex activity that requires the coordination of multiple structures. Currently there are different methods to evaluate this process, however, the ability to describe the behavior of structures in its three dimensions is limited. It has been shown that lingual movement is key for food bolus formation and propulsion to the oropharynx. Hence, lingual movement patterns have been identified through various techniques. The objective of this review is to report the different swallowing assessment techniques and identify the patterns established by each of them. A review of the literature was carried out in different databases, through a systematic search strategy. The key words "Deglutition", "Swallowing", "Movement Tracking", "evaluation" and "patterns" were used in English and Spanish. Unlimited studies without restriction on the date of publication, carried out in human subjects, in English, Spanish and Portuguese, were included. Different techniques were found to evaluate swallowing, videofluoroscopy, endoscopy, electromagnetic articulation, electromyography, computed tomography, manometry, electropalatography and ultrasound. Videofluoroscopy is one of the most popular methods used, this technique has attempted to describe patterns of movement during swallowing, as well as electromagnetic articulation. On the other hand, electromyography has described swallowing patterns, but in relation to the contraction and relaxation of certain muscle groups. There are different methods to assess swallowing. Each of the techniques has made it possible to elucidate this process better, nonetheless, a greater number of comparative studies is necessary to resolve doubts about the validity and effectiveness of the techniques described.

KEY WORDS deglutition, swallowing, movement tracking, patterns.

\section{REFERENCIAS BIBLIOGRÁFICAS}

Ardakani, F. E. Evaluation of swallowing patterns of the tongue using real-time B-mode sonography. J. Contemp. Dent. Pract., 7(3):6774,2006

Arteaga, J. P.; Olavarria, L. C.; Naranjo, D. B.; Elgueta, L. F. \& Espinóla, M. D. (2006). Cómo realizar una evaluación de deglución completa, eficaz y en corto tiempo. Rev. Otorrinolaringol. Cir. Cabeza Cuello, 66(1):13-22, 2006.

Bourdiol, P.; Mishellany-Dutour, A.; Peyron, M. A. \& Woda, A. Tonguemandible coupling movements during saliva swallowing. J. Oral Rehabil., 41(3):199-205, 2014. 
Chi-Fishman, G. \& Stone, M. A new application for electropalatography: swallowing. Dysphagia, 11(4):239-47, 1996.

Chi-Fishman, G.; Stone, M. \& McCall, G. N. Lingual action in normal sequential swallowing. J. Speech Lang. Hear. Res., 41(4):77185, 1998.

da Silva, A. P.; Lubianca Neto, J. F. \& Santoro, P. P. Comparison between videofluoroscopy and endoscopic evaluation of swallowing for the diagnosis of dysphagia in children. Otolaryngol. Head Neck Surg., 143(2):204-9, 2010.

Dodds, W. J.; Taylor, A. J.; Stewart, E. T.; Kern, M. K.; Logemann, J. A. \& Cook, I. J. Tipper and dipper types of oral swallows. A. J. R. Am. J. Roentgenol., 153(6):1197-9, 1989.

Engelke, W.; Jung, K. \& Knösel, M. Intra-oral compartment pressures: a biofunctional model and experimental measurements under different conditions of posture. Clin. Oral Investig., 15(2):165-76, 2010.

Ergun, G. A.; Kahrilas, P. J.; Lin, S.; Logemann, J. A. \& Harig, J. M. Shape, volume, and content of the deglutitive pharyngeal chamber imaged by ultrafast computerized tomography. Gastroenterology, 105(5):1396-403, 1993

Fattori, B.; Giusti, P.; Mancini, V.; Grosso, M.; Barillari, M. R.; Bastiani, L.; Molinaro, S. \& Nacci, A. Comparison between videofluoroscopy, fiberoptic endoscopy and scintigraphy for diagnosis of oro-pharyngeal dysphagia. Acta Otorhinolaryngol. Ital., 36(5):395-402, 2016.

Fernández Planas, A. M. La electropalatografía (EPG) en el estudio articulatorio del habla. El WinEPG de Articulate Instruments Ltd. Estud. Fon. Exp., 17:285-99, 2008.

Fuentes, R.; Engelke, W.; Flores, T.; Navarro, P.; Borie, E.; Curiqueo, A. \& Salamanca, C. Description of intraoral pressures on subpalatal space in young adult patients with normal occlusion. Int. J. Clin. Exp. Med., 8(7):11208-13, 2015

Fujii, N.; Inamoto, Y.; Saitoh, E.; Baba, M.; Okada, S.; Yoshioka, S.; Nakai, T.; Ida, Y.; Katada, K. \& Palmer, J. B. Evaluation of swallowing using 320-detector-row multislice CT. Part I: singleand multiphase volume scanning for three-dimensional morphological and kinematic analysis. Dysphagia, 26(2):99-107, 2011.

Gokyigit, M. C.; Pazarci, N. K.; Ercan, I.; Seker, S.; Turgut, S. \& Ertekin, C. Identification of distinct swallowing patterns for different bolus volumes. Clin. Neurophysiol., 120(9):1750-4, 2009.

Hiss, S. G.; Treole, K. \& Stuart, A. Effect of age, gender, and repeated measures on intraoral air pressure in normal adults. J. Voice, 15(2):159-64, 2001.

Jestrovic, I.; Coyle, J. L.; Perera, S. \& Sejdic, E. Functional connectivity patterns of normal human swallowing: difference among various viscosity swallows in normal and chin-tuck head positions. Brain Res., 1652:158-69, 2016.

Kieser, J.; Bolter, C.; Raniga, N.; Waddell, J. N.; Swain, M. \& Farland, G. Tongue-palate interactions during swallowing. J. Texture Stud., 42(2):95-102, 2011

Knösel, M.; Klein, S.; Bleckmann, A. \& Engelke, W. Tongue position after deglutition in subjects with habitual open-mouth posture under different functional conditions. Orthod. Craniofac. Res., 14(3):181-8, 2011

Kobayashi, M.; Asada, Y.; Matsubara, K.; Minami, K.; Suzuki, S.; Matsunaga, Y.; Haba, T.; Kawaguchi, A.; Daioku, T.; Toyama, H. \& Kato, R. Swallowing computed tomography: dose estimation in a phantom study conducted at various patient reclining angles. Radiat. Prot. Dosimetry, 178(1):87-94, 2018.

Langmore, S. E. Evaluation of oropharyngeal dysphagia: which diagnostic tool is superior? Curr. Opin. Otolaryngol. Head Neck Surg., 11(6): 485-9, 2003.

Langmore, S. E.; Schatz, K. \& Olson, N. Endoscopic and videofluoroscopic evaluations of swallowing and aspiration. Ann. Otol. Rhinol. Laryngol., 100(8):678-81, 1991.
Logemann, J. A.; Rademaker, A. W.; Pauloski, B. R.; Ohmae, Y. \& Kahrilas, P. J. (1998). Normal swallowing physiology as viewed by videofluoroscopy and videoendoscopy. Folia Phoniatr. Logop., 50(6):311-9, 1998.

MacAvoy, S. K.; Jack, H. C.; Kieser, J. \& Farella, M. Effect of occlusal vertical dimension on swallowing patterns and perioral electromyographic activity. J. Oral Rehabil., 43(7):481-7, 2016.

Nazar, M. G.; Ortega, T. A.; Godoy, M. A.; Godoy, M. J. M. \& Fuentealba, M. I. Evaluación fibroscópica de la deglución. Rev. Otorrinolaringol. Cir. Cabeza Cuello, 68(2):131-42, 2008.

Padrós Serrat, E. Cómo cuantificar las funciones y la postura en la consulta de ortodoncia. Rev. Ortod. Clin., 7(4):174-204, 2004.

Park, C. H.; Kim, D. K.; Lee, Y. T.; Yi, Y.; Lee, J. S.; Kim, K.; Park, J. H. \& Yoon, K. J. Quantitative analysis of swallowing function between dysphagia patients and healthy subjects using highresolution manometry. Ann. Rehabil. Med., 41(5):776-85, 2017.

Reynolds, J.; Carroll, S. \& Sturdivant, C. Fiberoptic endoscopic evaluation of swallowing: a multidisciplinary alternative for assessment of infants with dysphagia in the neonatal intensive care unit. Adv. Neonatal Care, 16(1):37-43, 2016.

Rocha, S. G.; da Silva, R. G. \& Berti, L. C. Qualitative and quantitative ultrasound analysis of oropharyngeal swallowing. CoDAS, 27(5):437-45, 2015.

Rosen, S. P.; Abdelhalim, S. M.; Jones, C. A. \& McCulloch, T. M. Effect of body position on pharyngeal swallowing pressures using high-resolution manometry. Dysphagia, 33(3):389-98, 2018.

Steele, C. M. \& Van Lieshout, P. H. The dynamics of lingualmandibular coordination during liquid swallowing. Dysphagia, 23(1):33-46, 2008

Steele, C. M. \& Van Lieshout, P. H. Use of electromagnetic midsagittal articulography in the study of swallowing. J. Speech Lang. Hear. Res., 47(2):342-52, 2004.

Steele, C. M. \& Van Lieshout, P. Tongue movements during water swallowing in healthy young and older adults. J. Speech Lang. Hear. Res., 52(5):1255-67, 2009.

Vaiman, M.; Eviatar, E. \& Segal, S. Surface electromyographic studies of swallowing in normal subjects: a review of 440 adults. Report 1. Quantitative data: timing measures. Otolaryngol. Head Neck Surg., 131(4):548-55, $2004 a$.

Vaiman, M.; Eviatar, E. \& Segal, S. Surface electromyographic studies of swallowing in normal subjects: a review of 440 adults. Report 2. Quantitative data: amplitude measures. Otolaryngol. Head Neck Surg., 131(5):773-80, 2004b.

Zhu, M.; Yu, B.; Yang, W.; Jiang, Y.; Lu, L.; Huang, Z.; Chen, S. \& Li, G. Evaluation of normal swallowing functions by using dynamic high-density surface electromyography maps. Biomed. Eng. Online, 16(1):133, 2017.

\section{Dirección para correspondencia:}

Prof. Dr. Ramón Fuentes Fernández

Centro de Investigación en Ciencias Odontológicas

Facultad de Odontología, Universidad de La Frontera

Av. Francisco Salazar 01145

Temuco

CHILE

E-mail: ramon.fuentes@ufrontera.cl

Recibido: 28-06-2018

Aceptado:15-09-2018 